\title{
Weisheitseinstellungen und Lebensbewältigung bei psychosomatischen Patienten
}

\section{Wisdom Attitudes and Coping In Life of Psychosomatic Patients}

Autoren

Michael Linden ${ }^{1}$, Barbara Lieberei², Nils Noack ${ }^{1}$

Institute

1 Research Group Psychosomatic Rehabilitation, Charité Universitätsmedizin Berlin

2 Heinrich-Heine-Klinik, Potsdam

\section{Schlüsselwörter}

Psychotherapie, Gerechtigkeit, Verbitterung, Lebensbelastung, Ressourcen

Key words

psychotherapy, belief in a just world, embitterment, burdens in life, Resources

eingereicht 17.05 .2018

akzeptiert 09.11.2018

Bibliografie

DOI https://doi.org/10.1055/a-0813-2040

Online-Publikation: 14.1.2019

Psychother Psych Med 2019; 69: 332-338

(c) Georg Thieme Verlag KG Stuttgart · New York

ISSN 0937-2032

Korrespondenzadresse

Prof. Dr. Michael Linden

Research Group Psychosomatic Rehabilitation

Charité University Medicine Berlin

Lichterfelder Allee 55

14513 Teltow/Berlin

michael.linden@charite.de

\section{ZUSAMMENFASSUNG}

Abstrakt Einleitung Weisheit ist eine menschliche Fähigkeit zur Bewältigung schwieriger Lebensprobleme, bzw. eine spezielle Art von Problemlösefähigkeit und damit eine wichtige Ressource in der Alltagsbewältigung. Sie findet in der psychologischen Forschung und auch der klinischen Praxis zunehmende Beachtung. Ziel der vorliegenden Studie ist die Untersuchung der Verteilung und Korrelationen von Weisheitseinstellungen bei psychosomatischen Patienten unter Anwendung der neu entwickelten 12-WDSkala.

Material und Methoden Mit der 12-WD-Skala werden 12 Dimensionen der Weisheit erfasst. Diese Skala wurde bei 202 unausgelesenen stationären Patienten einer psychosomatischen Rehabilitationsklinik als Teil der Routine-Eingangsuntersuchung eingesetzt. Es standen zusätzlich Daten aus der Basisdokumentation zu Verfügung. Zusätzlich füllten die Patienten die Differentielle Lebensbelastungsskala (DLB), die Allgemeine Gerechtigkeitsskala (GWAL) und eine Verbitterungs-Skala (PTED-Skala) aus. Aus der Basisdokumentation konnten soziodemografische und klinische Daten hinzugezogen werden.

Ergebnisse Die Verteilung des globalen Weisheitsscores entspricht annähernd einer Normalverteilung. Der Mittelwert liegt mit $M=4,50(S D=0,71)$ im positiven Bereich. Eine Faktorenanalyse ergab 3 Faktoren, die als „Nüchternheit und Realitätssinn“, „Gelassenheit“ und „Selbstbescheidung“ bezeichnet werden können und insgesamt 53,7\% der Gesamtvarianz erklärten. Es fanden sich positive Korrelationen des Weisheitsscores mit der allgemeinen Lebenszufriedenheit und dem Alter, negative mit Gerechtigkeitsansprüchen und Verbitterung, jedoch keine mit dem Bildungsgrad.

Diskussion Die Ergebnisse zeigen, dass die Patienten im Durchschnitt Weisheitseinstellungen eher bejahen. Eine höhere Zustimmung ist mit einer besseren Lebensbewältigung assoziiert. Weisheitskompetenzen bieten einen interessanten Ansatz in der psychotherapeutischen Behandlung von Menschen in schwierigen Lebenssituationen, wie dies typischerweise für psychosomatische Patienten gilt. Die 12-WD-Skala kann hierfür hilfreiche Informationen liefern.

\section{ABSTRACT}

Background Wisdom is a capacity, which is needed in coping with difficult problems in life. It can be understood as a special type of problem solving skill. It is getting growing recognition in psychology in general, and in psychotherapy and clinical practice in particular. Goal of the present study is to assess wisdom competencies and their correlates in psychosomatic patients, using for the first time the 12-WD-scale.

Method The 12-WD-Scale covers 12 wisdom dimensions. As part of their routine intake assessment, 202 unselected inpatients of a department of psychosomatic medicine filled in the 12-WD-scale together with the differential life burden scale, the global belief in a just world scale and the posttraumatic embitterment scale. Additional patient and clinical data could be taken from the routine data. 
Results Wisdom scores showed a normal distribution. The mean was in the positive range $(4.50, S D=0.71)$. A factor analysis showed three factors (sobriety, serenity, modesty), explaining $53.7 \%$ of the total variance. There were positive correlations of the wisdom score with life satisfaction and age, negative correlations with beliefs in justice and embitterment, but not with formal education.
Discussion The results of the 12-WD-Scale show that psychosomatic patients appreciate wisdom attitudes and that this is associated with better coping in life. Wisdom competencies are an interesting field in psychotherapy when patients are burdened by difficult situations in life. The 12-WD-scale can provide helpful information in this regard.

\section{Einleitung}

Im der der Entwicklungspsychologie und Psychologie der Lebensspanne ist das Thema „Weisheit“" zu einem eigenen psychologischen Forschungsschwerpunkt geworden [1-10]. Weisheit ist definiert als Expertise im Umgang mit schwerwiegenden und unveränderbaren Problemen des Lebens, die sich insbesondere bei Fragen der Lebensgestaltung, Lebensplanung und Lebensdeutung zeigt und eine Adaptation an komplexe situative-, umweltbezogene-, und personenbezogene Anforderungen ermöglicht [1,8]. Sie ist jedem Menschen zu eigen und ist eine Fähigkeit, mittels der Widersprüche in schwierigen Situationen zu bewältigen oder Konsequenzen einer Handlung für sich selbst und andere realistisch abgewogen werden können [11]. Weisheit ist nötig, um in schwierigen Situationen eine Balance zwischen intrapersonellen, interpersonalen und institutionellen Interessen herzustellen. Ein wichtiges Kriterium in der Definition weisheitsbezogenen Wissens und Urteilens ist die existenzielle Dimension bezogen auf die fundamentale Pragmatik des Lebens, die das Wissen um lebenslange Veränderungen bzw. Entwicklungen, die Begrenztheit des eigenen Wissens und der körperlichen Existenz, die soziale Vernetztheit des Lebens, die Berücksichtigung von unveränderbaren, unverständlichen Grundgegebenheiten (z. B. Geburt und Tod) sowie konkrete Kenntnisse über Lebensaufgaben und Lebensziele mit einschließt. Als Ressource zur Konflikt- bzw. Stressbewältigung steht Weisheit rigiden, dogmatischen und unflexiblen Denkweisen gegenüber [11].

Zusammenfassend kann Weisheit als spezielle Form einer Problemlösefähigkeit verstanden werden. Sie ist eine wichtige Ressource nicht nur im Umgang mit schwierigen Lebenssituationen, sondern auch in der Alltagsbewältigung und sogar Alltäglichkeiten, wie z. B. beim Einkaufen im Supermarkt. Auch dort stellen sich Dilemmata, bspw., ob man eher ein hochwertiges aber teures Produkt oder ein preiswertes No-Name-Produkt kaufen soll. Es gibt keine eindeutige oder richtige Antwort, sondern es sind mehrdimensionale, komplexe Überlegungen erforderlich, ohne je Eindeutigkeit erreichen zu können. Weisheit ist somit ein allgemeiner Resilienzfaktor und ist ähnlich wie Selbstwirksamkeit oder soziale Unterstützung für die Bewältigung von Lebensanforderungen und -belastungen aller Art hilfreich bzw. nötig ist. Von daher ist es eine psychologische Dimension mit unmittelbarer Bedeutung für die Psychotherapie [13]. Es konnte gezeigt werden, dass Weisheit, analog zur Selbstsicherheit oder anderen psychischen Kompetenzen, lernbar und trainierbar ist. Damit ergab sich die Möglichkeit, die Förderung von Weisheit auch zum Psychotherapieziel zu machen. Von Linden und Kollegen [14, 15] wurden unter dem Titel der „Weisheitstherapie“ hierfür einige psychotherapeutische Strategien beschrieben und auch in ersten Studien auf Wirksamkeit untersucht.
Weisheit ist ein mehrdimensionales Konstrukt [12], so wie auch soziale Kompetenz. Fasst man die verschiedenen Definitionsansätze in der Weisheitsforschung zusammen, dann lassen sich im Wesentlichen 12 Subdimensionen nennen [4] ( $\triangleright$ Tab. 1). Weisheit in Bezug auf den „Blick auf die Welt“ schließt die Kompetenzen „Fakten- Problemlösewissen“, „Kontextualismus“ und „Wertrelativismus“ ein. Dimensionen mit „Blick auf andere Menschen“ sind „Perspektivenübernahme“ und „Empathie“. Zum „Blick auf die eigene Person“ gehören „Problem- und Anspruchsrelativierung“, „Selbstrelativierung“ und „Selbstdistanz“. Der „Blick auf das eigene Erleben“ schließt die „Emotionswahrnehmung und Akzeptanz“ sowie „Emotionale Serenität im Sinne emotionaler Gelassenheit und Steuerungsfähigkeit sowie Humor“ mit ein. Der „Blick auf die Zukunft“ umfasst „Ungewissheitstoleranz“ und „Nachhaltigkeit“. Auch wenn die einzelnen Dimensionen nicht immer eindeutig trennscharf sind, so ermöglicht die Abgrenzung der verschiedenen Konstituenten der Weisheit unmittelbar auch Trainings- und Therapieinterventionen.

Es gibt eine Reihe von Instrumenten zur Operationalisierung, Erfassung und Quantifizierung von Weisheit. Die „Practical Wisdom Scale“ (PWS) von Wink und Helson [16] ist eine Adjektiv-Checkliste mit vierzehn weisheitsbezogenen (z. B. „reflective“, „clearthinking“, „mature“) und vier weisheitsfernen Eigenschaften (z. B. „intolerant“, „reckless“ und „shallow“). Das „Transcendent Wisdom Rating“ (TWR) [16] ist eine inhaltsanalytische Fremdbeurteilung, der frei formulierten Antworten, die Probanden auf die Frage geben: „Viele Menschen hoffen mit dem Alter weiser zu werden. Können Sie ein Beispiel nennen, wie Sie selbst zu mehr Weisheit kamen?". Die Antworten werden von Experten auf einer 5-stufigen Skala beurteilt. Die 3-dimensionale Weisheitsskala (3-d WS) von Ardelt [17] erfasst anhand von 39 Items 3 Komponenten, d. h. eine kognitive Dimension (14 Items; „I am hesitant about making important decisions after thinking about them"), eine reflektive Dimension (12 Items; „I always try to look at all sides of a problem”), und eine affektive Komponente (13 Items; „,sometimes I don't feel very sorry for other people when they are having problems"). Die Self-Assessed Wisdom Scale (SAWA) $[18,19]$ soll Weisheit als „competence in, intention to and application of critical life experience to facilitate the optimal development of self and others" beschreiben $[19,20]$. Die Erfassung von Weisheit erfolgt über 5 miteinander verknüpfte Persönlichkeitsdimensionen, d. h. „experience“, die Lebenserfahrung mit schwierigen und die Moral betreffenden Lebensereignissen, „emotion regulation“, die Möglichkeit starke und subtile Emotionen zu empfinden und damit umgehen zu können, „reminiscence and reflectiveness“, die Fähigkeit und Motivation über das Leben intensiv nachzudenken, sich mit der eigenen Identität auseinanderzusetzen und mit Problemen umgehen zu kön- 
- Tab. 1 Weisheitskompetenzen.

\section{A. Blick auf die Welt}

1. Fakten- und Problemlösewissen generelles und spezifisches Wissen um Probleme und Problemkonstellationen, was Probleme konstituiert und welche Möglichkeiten der Problemlösung es gibt.

2. Kontextualismus Wissen um die zeitliche und situative Einbettung von Problemen und die zahlreichen Umstände, in die ein Leben eingebunden ist.

3. Wertrelativismus

Wissen um die Vielfalt von Werten und Lebenszielen und die Notwendigkeit, jede Person innerhalb ihres Wertesystems zu betrachten, ohne dabei eine kleine Anzahl universeller Werte aus dem Auge zu verlieren.

\section{Blick auf andere Menschen}

4. Perspektivwechsel

Fähigkeit zum Beschreibung eines Problems aus der Sichtweise verschiedener daran beteiligter Personen.

5. Empathie

Fähigkeit, sich in das emotionale Erleben einer anderen Person hineinversetzen zu können.

\section{Blick auf die eigene Person}

6. Problem- und Anspruchsrelativierung

Fähigkeit zur Bescheidenheit und zur Akzeptanz, dass die eigenen die eigenen Probleme im Vergleich mit vielen Problemen der Welt nicht so wichtig genommen werden dürfen.

7. Selbstrelativierung

Fähigkeit zu akzeptieren, dass man selbst nicht immer am wichtigsten ist und vieles nicht nach dem eigenen Willen läuft oder sich an den eigenen Interessen orientiert.

8. Selbstdistanz

Fähigkeit, die Wahrnehmungen und Bewertungen der eigenen Person aus der Sicht anderer Menschen erkennen und nachvollziehen zu können.

\section{Blick auf das eigene Erleben}

9. Emotionswahrnehmung und Emotionsakzeptanz Fähigkeit zur Wahrnehmung und Akzeptanz eigener Gefühle.

10. Emotionale Serenität und Humor Fähigkeit zur emotionalen Ausgeglichenheit, zur Steuerung der eigenen Emotionen nach Situationserfordernissen sowie die Fähigkeit, sich selbst und die eigenen Schwierigkeiten mit Humor zu betrachten.

\section{Blick auf die Zukunft}

11. Ungewissheitstoleranz

Wissen um die dem Leben inhärente Ungewissheit bezüglich Vergangenheit, Gegenwart und Zukunft.

12. Nachhaltigkeit Wissen um negative und positive Aspekte jedes Geschehens und Verhaltens, sowie kurz- und langfristige Konsequenzen, die sich auch widersprechen können.

nen, „openess“, das Interesse an neuen Möglichkeiten, Perspektiven und Problemlösestrategien sowie „humor“, die Fähigkeit auch in ernsten Situationen komische Aspekte zu sehen. Die Autorin berichtet eine akzeptable Reliabilität über Cronbach's Alpha von 0,78 für die Gesamtskala. Im „Adult Self-Transcendence Inventory“
(ASTI) [21] wird Weisheit mit 18 Items operationalisiert durch 4 Eigenschaften „Selbsterkenntnis (,self-knowledge“), „Distanziertheit („detachment“), „Integration“ (,integration“) und „Selbsttranszendenz“ („self-transcendence“). Selbsttranszendenz wird als zentrale Komponente der Weisheit angesehen im Sinne einer Fähigkeit zur Entwicklung von Spiritualität, der Eigenständigkeit des Selbst und einem Gefühl von Verbundenheit mit der Vergangenheit und Zukunft, welche als angeborenes Streben nach der Ergründung eines Lebenssinns gesehen wird. Um die vorgenannten 12 Weisheitsdimensionen systematisch erfassen zu können, wurde die 12-WD-Skala entwickelt, die im Folgenden näher beschrieben wird und erstmalig in der vorliegenden Untersuchung verwendet wurde.

Trotz der grundsätzlichen Bedeutung von Weisheit für die Lebensbewältigung und trotz der Möglichkeit, Weisheit im Sinne eines Resilienzfaktors trainieren und psychotherapeutisch nutzen zu können, gibt es bislang nur ansatzweise eine systematische Übertragung dieser grundlagenwissenschaftlichen Forschungsergebnisse in die klinische Praxis. In einem ersten Schritt interessiert die Frage, wie sich Weisheit in klinischen Populationen verteilt und welche Zusammenhänge zwischen dem Ausmaß subjektiver Weisheitseinstellungen und der Lebensbewältigung zu finden sind. Dies wurde in der vorliegenden Studie u. W. erstmals unter Einsatz der 12-WD-Skala an einer klinischen Stichprobe untersucht.

\section{Methode}

\section{Stichprobe und Untersuchungsdurchführung}

Die Untersuchung wurde an einer Psychosomatischen Rehabilitationsklinik (Heinrich Heine Klinik, Potsdam) durchgeführt. Die Klinik behandelt Patienten aus dem gesamten Spektrum psychischer Störungen, mit Schwerpunkt auf den affektiven Erkrankungen und Persönlichkeitsstörungen. Patienten werden über Vermittlung der Renten- und Krankenversicherungen zugewiesen wegen Problemen mit der Arbeits- und Erwerbsfähigkeit.

Die Fragebögen, die in die vorliegende Untersuchung eingingen, wurden den Patienten im Rahmen der routinemäßigen Eingangsuntersuchung vorgelegt. Es erfolgte keine spezielle Patientenselektion und es gab keine Drop-outs. Die Datenerhebung fand in einem Zeitraum von 2 Monaten statt.

\section{Instrumente}

Als Indikator der allgemeinen Lebensbewältigung wurde die „Differentielle Lebensbelastungsskala“ (DLB-Skala) [22] eingesetzt. Sie erfasst mit 17 Items über alle wichtigen Lebensbereiche hinweg das subjektive Belastungserleben. Es wird vorgegeben: „Wenn ich an das Thema XY (z. B. Familie) denke, sind meine Gefühle (..)“, gefolgt von dem Rating: „1 = sehr negativ“ bis „6 = sehr positiv“ getroffen. Es kann ein Durchschnittsscore über alle Items hinweg gebildet werden, der den Grad des allgemeinen Stresserlebens bzw. der erfahrenen Lebensbelastung repräsentiert.

Als Dimension, für die ein direkter Zusammenhang mit unzureichenden Weisheitskompetenzen angenommen wird, wurde die PTED Skala (Posttraumatic Embitterment Scale) [23] verwendet. Sie erfasst anhand von 20 Items belastungsabhängige Verbitterungsreaktionen. Der Einleitungssatz lautet: „In den letzten Jahren hatte ich ein einschneidendes Lebensereignis zu verkraften (..)“ 
und darauf folgend z. B. „(..), das in mir Gedanken an Rache auslöst“. Ein PTED Score von $\geq 2,5$ spricht für eine klinisch bedeutsame Verbitterungstendenz [23].

Die „Allgemeine Gerechte-Welt-Skala“ (GWAL) [24] ist ein Selbstbeurteilungsbogen mit 6 Items zur Erfassung der Gerechtigkeitsüberzeugungen einer Person. Dieses Konstrukt hat einen unmittelbaren theoretischen Zusammenhang zu Weisheit. Ein Beispielitem lautet: „Ich glaube, dass die Leute im Großen und Ganzen das bekommen, was ihnen gerechterweise zusteht“. Die Ratings werden auf einer 6-stufigen Skala von „1 = stimmt genau“ bis „6 = stimmt überhaupt nicht“ eingeschätzt. Ein Skalenwert kann über den Mittelwert bestimmt werden.

Die 12-WD-Skala (Skala zur Erfassung von 12 Weisheitsdimensionen) ist eine Neuentwicklung in Anlehnung an die vorgenannten 12 Weisheitsdimensionen [4]. Aus einem Itempool von zunächst 237 Items wurden mittels der Delphi-Methode [25] 36 Items ausgewählt, die den theoretischen Definitionen der Weisheitsdimensionen entsprachen. Im Anschluss wurden mittels konfirmatorischer Faktorenanalyse 12 Items extrahiert, indem für jede Dimension das Item mit der höchsten semipartiellen Korrelation mit dem jeweiligen Faktor ausgewählt wurde. Die finale Version der 12-WD-Skala ist in > Tab. 2 dargestellt. Es wird als Instruktion vorgegeben: „Im Folgenden finden
Sie ganz unterschiedliche Aussagen und Leitsätze dazu, wie Menschen auf Schwierigkeiten und Lebensbelastungen reagieren können. Sie werden wahrscheinlich einigen Aussagen sehr zustimmen und andere stark ablehnen. Bei wieder anderen sind Sie vielleicht unentschieden. Nehmen Sie bitte zu jeder der folgenden Aussagen Stellung und entscheiden Sie, inwieweit jede auf Sie ganz persönlich zutrifft“. Die Items werden auf einer 6-stufigen Likert-Skala (1 = stimmt überhaupt nicht; 2 = stimmt weitgehend nicht; 3 = stimmt eher nicht; 4 = stimmt ein wenig; 5 = stimmt weitgehend; 6 = stimmt genau) beurteilt. Die Items fragen nach generellen Einstellungen zu Aussagen wie „Nach meiner Meinung soll jeder auf seine Art glücklich werden “. Der Fragebogen erfasst, mit theoretischer Referenz zu den 12 Subkomponenten, weisheitsbezogene Einstellungen, was nicht unmittelbar mit weisheitskompetentem Verhalten gleichgesetzt werden kann.

\section{Ergebnisse}

Es standen Fragebögen von 202 unausgelesenen Patienten zur Verfügung. Davon waren 71,8\% ( $=145)$ weiblich, das Durchschnittsalter betrug 50 Jahre $(M=50,02 ; S D=9,52), 21,6 \%(N=43)$ waren ledig, 58,3\% ( $N=116)$ verheiratet, 17,6\% $(N=35)$ geschieden und $2,5 \%(N=5)$ verwitwet. $2 \%(N=4)$ hatten keinen Schulabschluss,

Tab. 2 Die 12-WD-Skala.

\begin{abstract}
Im Folgenden finden Sie ganz unterschiedliche Aussagen und Leitsätze dazu, wie Menschen auf Schwierigkeiten und Lebensbelastungen reagieren können. Sie werden wahrscheinlich einigen Aussagen sehr zustimmen und andere stark ablehnen. Bei wieder anderen sind Sie vielleicht unentschieden. Nehmen Sie bitte zu jeder der folgenden Aussagen Stellung und entscheiden Sie inwieweit jede auf Sie ganz persönlich zutrifft
\end{abstract}

\begin{tabular}{|c|c|c|c|c|c|}
\hline $\begin{array}{l}\text { Stimmt } \\
\text { überhaupt } \\
\text { nicht }\end{array}$ & $\begin{array}{l}\text { Stimmt } \\
\text { weitgehend } \\
\text { nicht }\end{array}$ & $\begin{array}{l}\text { Stimmt } \\
\text { eher nicht }\end{array}$ & $\begin{array}{l}\text { Stimmt ein } \\
\text { wenig }\end{array}$ & $\begin{array}{l}\text { Stimmt } \\
\text { weitgehend }\end{array}$ & $\begin{array}{l}\text { Stimmt } \\
\text { genau }\end{array}$ \\
\hline 1 & 2 & 3 & 4 & 5 & 6 \\
\hline 1 & 2 & 3 & 4 & 5 & 6 \\
\hline 1 & 2 & 3 & 4 & 5 & 6 \\
\hline 1 & 2 & 3 & 4 & 5 & 6 \\
\hline 1 & 2 & 3 & 4 & 5 & 6 \\
\hline 1 & 2 & 3 & 4 & 5 & 6 \\
\hline 1 & 2 & 3 & 4 & 5 & 6 \\
\hline 1 & 2 & 3 & 4 & 5 & 6 \\
\hline 1 & 2 & 3 & 4 & 5 & 6 \\
\hline 1 & 2 & 3 & 4 & 5 & 6 \\
\hline 1 & 2 & 3 & 4 & 5 & 6 \\
\hline 1 & 2 & 3 & 4 & 5 & 6 \\
\hline
\end{tabular}

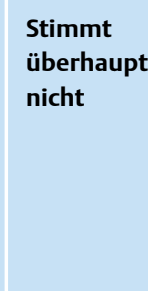

1. Bevor ich auf ein Problem reagiere, ist es mir wichtig, erst einmal zu verstehen, worin das Problem besteht

2. Was gut oder schlecht ist, hängt wesentlich von den Rahmenbedingungen ab

3. Nach meiner Meinung soll jeder auf seine Art glücklich werden

4. Für mich ist es interessant sich zu überlegen, was andere zu einem Thema denken

5. Ich versuche immer mitzufühlen, wie mein Gegenüber sich fühlt

6. Wenn man zufrieden ist mit dem, was man hat, dann geht es einem besser, als wenn man dem nachweint, was man nicht hat

7. Ich versuche mich nach Möglichkeit nicht so ernst zu nehmen

8. Ich kann nicht erwarten, dass andere mich mögen, wenn ich mich nicht entsprechend benehme

9. Ich kann nicht verlangen, immer nur guter Stimmung zu sein

10. Ich versuche mich nach Möglichkeit nicht aufzuregen, denn man hat ja nicht davon, wenn man sich selbst aufregt

11. Ich gehöre zu den Menschen, die sagen, es kommt, wie es kommt

12. Ich sehe Krisen immer auch als Chance für die Zukunft 
22,5\% ( $N=51)$ einen Haupt- bzw. Sonderschulabschluss, 34,2\% $(\mathrm{N}=69)$ eine mittlere Reife bzw. eine abgeschlossene Berufsausbildung, 17,3\% ( $=35)$ das Abitur und 21,3\% $(N=43)$ einen universitären Abschluss. Die Erstdiagnosen bei Aufnahme waren affektive Störungen (F30-F39) mit 64,9\% (N=131), Angst-, Belastungs- und somatoforme Störungen mit 28,7\% ( $\mathrm{N}=58)$, Verhaltensauffälligkeiten mi körperlichen Störungen und Faktoren (F50-F59) mit 1,0\% $(N=2)$, Persönlichkeitsstörungen (F60-F69) mit 1,5\% ( $N=3)$, Verhaltens- und emotionale Störungen mit Beginn in der Kindheit und Jugend (F90-F99) mit 1,0\% (N=2), Schilddrüsen Unterfunktion (E03.9) mit 1,0\% (N=2) essentielle primäre Hypertonie (I10.00) mit $0,5 \%(N=1)$, Reizdarmsyndrom (K58.0) mit 0,5\% ( $N=1)$ und Probleme mit Bezug auf Schwierigkeiten bei der Lebensbewältigung (Z73) mit 0,5\% ( $\mathrm{N}=1)$.

Der globale Weisheitsscore ist annähernd normalverteilt, mit einem Mittelwert von $M=4,50(S D=0,71)$, einem Minimum von 1,24 und einem Maximum von 6,0. Im Durchschnitt haben nur $7,1 \%$ der Patienten $(N=14)$ einen Gesamtwert von $<3,5$, was als „unweise“ bezeichnet werden könnte, da sie häufiger den Items nicht zugestimmt haben bzw. ein Durchschnittsrating von unter „4 = stimmt ein wenig“ getroffen haben. > Abb. 1. zeigt die Prozentwerte, mit denen die einzelnen Items der Weisheitsskala bejaht oder abgelehnt wurden. Ungewissheitstoleranz, Selbstrelativierung, emotionale Serenität und Nachhaltigkeit sind die Dimensionen, denen vergleichsweise am häufigsten nicht zugestimmt wurde, d. h. von etwa 15 bis $20 \%$ der Patienten.

Cronbach's Alpha beträgt $\alpha=0,81$. Eine oblique Faktorenanalyse mit Promax-Rotation erbrachte 3 Komponenten, die jeweils durch 4 Items mit hohen Ladungen abgebildet werden. Der erste Faktor wird durch die Items des Fakten- und Problemlösewissens, der Nachhaltigkeit, der emotionalen Serenität und Unsicherheitstoleranz gebildet und klärt 33,41 \% der Gesamtvarianz. Man könne dies als „Nüchternheit und Realitätssinn“ zusammenfassen. Der zweite Faktor umfasst Wertrelativismus, Anspruchsrelativierung, Kontextualismus, sowie Emotionswahrnehmung und -akzeptanz mit 10,58\% der Gesamtvarianz, was als „Gelassenheit“ be- zeichnet werden kann. Der dritte Faktor umfasst Selbstdistanz, Perspektivwechsels, Empathie und Selbstrelativierung mit 9,72\% der Gesamtvarianz, was als „Selbstbescheidung“ benannt werden kann. Die 3 Komponenten decken 53,71\% der Gesamtvarianz auf. Interkorrelation zwischen Faktor 1 und Faktor 2 beträgt $r=0,33$, zwischen Faktor 1 und Faktor $3 r=0,43$ und zwischen Faktor 2 und Faktor $3 r=0,31$.

Es fanden sich signifikant positive Korrelationen des globalen Weisheitsscores mit Indikatoren der Lebensbewältigung (DLB-Skala) und Konstrukten, von denen theoretisch ein enger Bezug zur Weisheit anzunehmen ist (PTED-Skala, GWAL-Skala). Für die DLBSkala fand sich, dass ein höherer Weisheitsscore mit höherer allgemeiner Lebenszufriedenheit korreliert $(r=0,23 ; p=0,001)$. Es fanden sich Zusammenhänge mit der GWAL-Skala $(r=0,16 ; p=0,03)$, d. h. je höher der Weisheitsscore, desto weniger bestehen die Patienten auf Gerechtigkeit. Es fand sich eine negative Korrelation mit der PTED-Skala $(r=-0,15 ; p=0,04)$, d. h. je höher der Weisheitsscore, desto weniger klagen die Patienten über Verbitterung. Schließlich findet sich auch eine positive Korrelation mit dem Alter $(r=0,15 ; p=0,04)$. Es finden sich keine signifikanten Zusammenhänge mit dem Bildungsgrad der Patienten $(r=0,05$; $p=0,47)$, dem Geschlecht oder den Hauptdiagnosegruppen.

\section{Diskussion}

Die Unterstützung von Patienten mit schwierigen und häufig nicht wieder gut zu machenden Lebensproblemen gehört zu den häufigen Aufgabenstellungen in der Psychotherapie [26, 27]. Obwohl nach der psychologischen Grundlagenforschung Weisheit in solchen Fällen als wichtige Ressource anzusehen ist, gibt es unseres Wissens bislang keine Daten zu Weisheitseinstellungen und -kompetenzen psychosomatischer Patienten. Insofern können wir auch keine Vergleiche mit anderen Studien anstellen.

Die neu entwickelte 12-WD-Skala ist ein Instrument, das den Stand der psychologischen Grundlagenforschung zu Weisheit in ein kurzes Messinstrument übersetzen soll, womit ein Screening-

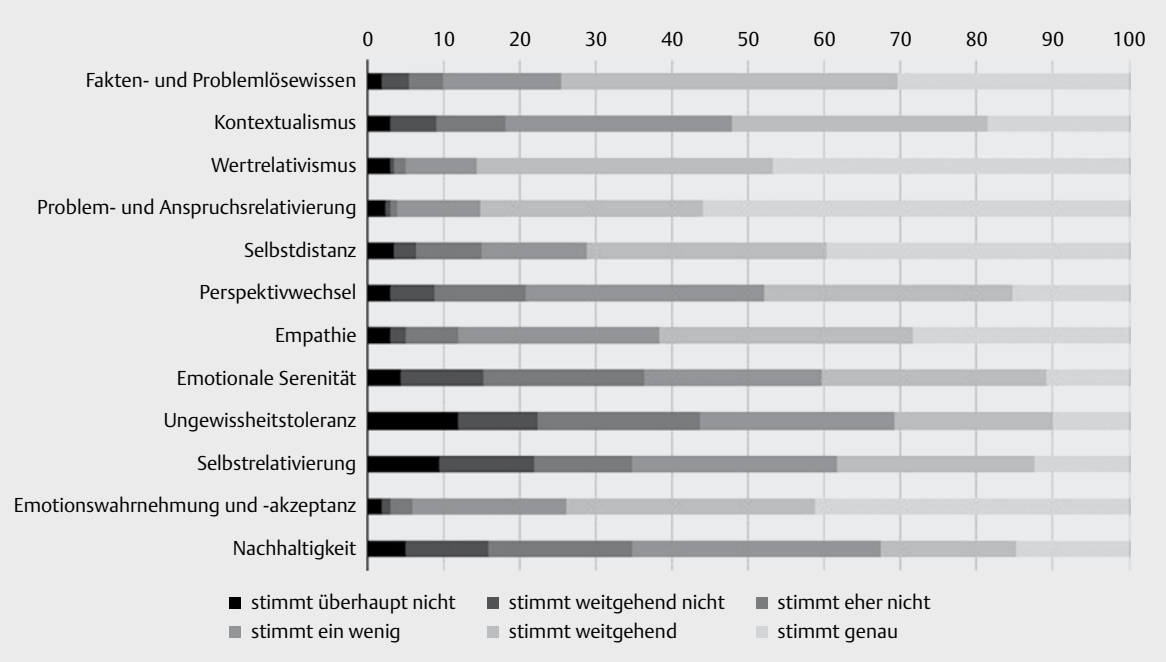

Abb. 1 Verteilung der Ablehnungs- bzw. Zustimmungshäufigkeit (\% der Patienten) je Item der 12-WD-Skala. 
verfahren zur Verfügung steht. Die Items der Skala wurden in einem ersten Schritt theoretisch deduktiv abgeleitet und dann faktorenanalytisch basiert ausgewählt.

Das hohe Cronbach-Alpha spricht zunächst einmal für eine hohe Interkorrelation aller Skalenitems. Hinzu kommt, dass die Verteilung des Summenscores eine annähernde Normalverteilung zeigt. Die mit der 12-WD-Skala erfassten Weisheitseinstellungen können somit als Globalkonstrukt interpretiert werden.

Dennoch ist auch eine Betrachtung von Subdimensionen von Interesse. Die Faktorenanalyse fasst die 12 Items in drei Subdimensionen zusammen, d. h. „Nüchternheit und Realitätssinn“ (Fakten- und Problemlösewissen, Nachhaltigkeit, emotionale Serenität, Unsicherheitstoleranz), „Gelassenheit“ (Wertrelativismus, Anspruchsrelativierung, Kontextualismus, Emotionswahrnehmung und -akzeptanz) und „Selbstbescheidung“ (Selbstdistanz, Perspektivwechsels, Empathie, Selbstrelativierung). Diese Gruppierungen haben eine hohe Augenschein-Validität und lassen sich gut in die verschiedenen Weisheitstheorien und -konzepte integrieren.

Von Interesse ist schließlich auch eine Betrachtung der Einzelitems. Es gibt Unterschiede in den Zustimmungsraten mit Ungewissheitstoleranz, Selbstrelativierung, emotionale Serenität und Nachhaltigkeit als die problematischsten Dimensionen. Es ist zu diskutieren, inwieweit es sich hierbei um krankheitsspezifische Auswirkungen handelt. Eine mangelnde Ungewissheitstoleranz ist beispielsweise bei Angsterkrankungen ein wichtiger pathogenetischer Faktor $[28,29]$. Emotionale Instabilität ist geradezu ein Primärmerkmal vieler psychischer Störungen [30, 31]. Und auch die Unfähigkeit von sich selbst Abstand zu finden, kennzeichnet viele einschlägige Erkrankungen und ist i.S. der Achtsamkeit geradezu ein vorrangiges Therapieziel [32]. Es wird weiterer Forschung bedürfen, um zwischen Psychopathologie und Weisheit zu unterscheiden. Vorstellbar ist auch, dass Weisheitseinstellungen, so wie bspw. auch Selbstsicherheit leiden können, wenn eine psychische Krankheit auftritt.

Große Übereinstimmung mit theoretischen Erwartungen zeigen die Korrelate der Weisheitseinstellungen. Wie aus der bisherigen Forschung zur Weisheit zu erwarten, zeigen Menschen mit höheren Weisheitsscores mehr Zufriedenheit mit ihrer Gesamtlebenssituation und weniger Verbitterung. Es findet sich auch eine negative Korrelation mit Gerechtigkeitsansprüchen, was i.S. von „Realitätssinn“, „Gelassenheit“ und „Selbstbescheidung“ ermöglicht, auch mit Ungerechtigkeit und Widrigkeiten im Leben besser umzugehen mit der Folge geringerer Lebensbelastungen und Verbitterung.

Es zeigt sich der Literatur entsprechend eine Korrelation mit Alter als Ausdruck der wachsenden Lebenserfahrung und Konfrontationen mit weisheitsfördernden Situationen [7, 33, 34]. Ebenfalls in Übereinstimmung mit der Literatur steht, dass kein Zusammenhang mit dem Geschlecht oder der formalen Bildung gefunden wurde. Lebenserfahrungen sind etwas Anderes als Buchwissen.

Von Interesse ist schließlich auch das allgemeine Niveau der Weisheitseinstellungen. Der Mittelwert von 4,5, bei einem Rating von 4 = stimmt ein wenig und 5 = stimmt weitgehend, spricht dafür, dass die Mehrzahl der Menschen in der Tendenz eher positive allgemeine Weisheitseinstellungen haben. Dennoch gibt es $7 \% \mathrm{mit}$ Hinweisen auf deutliche Weisheitseinschränkungen. Zukünftige Untersuchungen sollten detaillierter klären, was dies für Personen sind und womit dies zusammenhängt.
Die Ergebnisse müssen aber zunächst einmal auch mit gewisser Vorsicht interpretiert werden. Die statistischen Zusammenhänge zwischen Weisheit, Lebenszufriedenheit, Verbitterung, Gerechtigkeitserleben und Alter sind zwar signifikant, liegen aber eher im niedrigen Bereich. Ein weiteres Problem bei der Messung von Weisheit mit Selbstberichtsskalen ist, dass sie einem Bias der sozialen Erwünschtheit unterliegen und dies insbesondere auf das zur Rede stehende Konstrukt. In einer Studie von Bluck und Glück [35] wurde gezeigt, dass weise Menschen eher selbstkritisch sind. Vor diesem Hintergrund könnte man annehmen, dass weise Menschen sich selbst als eher weniger weise beurteilen. Es stellt sich die Frage, ob die Abfrage von Einstellungen dem Konstrukt adäquat ist und ob nicht eher eine verhaltensorientierte Erhebung erforderlich ist. Weitere Einschränkungen sind, dass die Daten ausschließlich aus einer Querschnitterhebung an einer psychosomatischen Population stammen. Es stehen zudem nur begrenzte Zusatz-Informationen zur Verfügung. Es sind weitere Studien erforderlich zur Replikation der Ergebnisse an anderen Populationen mit Wiederholungserhebungen und Beobachtungsdaten.

\section{FAZIT FÜR DIE PRAXIS}

Die vorliegende Studie ist eine erste explorative Untersuchung mit der neu entwickelten 12-WD-Skala. Aus den Daten kann abgeleitet werden, dass die mit der 12-WDSkala erfassten Weisheitseinstellungen sich, so wie viele andere psychologische Dimensionen normal verteilen und dass es theoriekonforme Zusammenhänge mit Indikatoren der Lebensbewältigung gibt. Dies bestätigt, dass Weisheit in der Untersuchung wie der Behandlung psychosomatischer Patienten angemessene Aufmerksamkeit zukommen sollte.

\section{Interessenkonflikt}

Die Autoren geben an, dass kein Interessenskonflikt besteht.

\section{Literatur}

[1] Baltes PB, Smith J. Weisheit und Weisheitsentwicklung: Prolegomena zu einer psychologischen Weisheitstheorie. Z Entwicklungspsychol Pädagog Psychol 1990; 22: 95-135

[2] Baltes PB, Staudinger UM. The search for a psychology of wisdom. Cur Dir Psychol Sci 1993; 2: 75-80

[3] Baltes PB, Glück J, Kunzmann U. Wisdom: its structure and function in regulating successful life span development. In: Snyder CR, Lopez SJ, (Hrsg.). Handbook of positive psychology. Oxford: Oxford University Press; 2002: 327-347

[4] Baumann K, Linden M., (Hrsg.). Weisheitskompetenzen und Weisheitstherapie - Die Bewältigung von Lebensbelastungen und Anpassungsstörungen. Lengerich: Pabst; 2008

[5] Dittmann-Kohli F, Baltes PB. Toward a neofunctionalist conception of adult intellectual development: Wisdom as a prototypical case of intellectual growth. In: Alexander C, Langer E, (Hrsg.). Higher Stages of human development: Perspectives on adult growth. New York: Oxford University Press; 1990: 54-78 
[6] Dixon RA, Baltes PB. Toward Life-span research on the functions and pragmatocs of intelligence. In: Sternberg RJ, Wagner RK, (Hrsg.). Practical intelligence: nature and origins of competence in the every-day world. New York: Cambridge University Press; 1986: 203-234

[7] Glück J., (Hrsg.). Weisheit. Die 5 Prinzipien des gelingenden Lebens. München: Kösel-Verlag; 2016

[8] Staudinger UM, Baltes PB. Weisheit als Gegenstand psychologischer Forschung. Psychol Rundsch 1996; 47: 57-77

[9] Staudinger U. Lebenserfahrung, Lebenssinn und Weisheit. In: Filipp $\mathrm{S}-\mathrm{H}$, Staudinger UM, (Hrsg.). Entwicklungspsychologie des mittleren und höheren Erwachsenenalters. Göttingen: Hogrefe; 2005: 739-761

[10] Staudinger UM, Dörner J. Weisheit, Einsicht und Persönlichkeit. In: Brandstädter J, Lindenberger U, (Hrsg.). Entwicklungspsychologie der Lebensspanne. Stuttgart: Kohlhammer; 2007

[11] Linden M., (Hrsg.). Verbitterung und Posttraumatische Verbitterungsstörung. Göttingen: Hogrefe; 2017

[12] Linden M. Wisdom and wisdom psychotherapy in coping with stress. In: Koh KB, (Hrsg.). Somatization and psychosomatic symptoms. New York: Springer; 2013: 273-281

[13] Pfeifer S., (Hrsg.). Weisheit als Ressource in der Psychotherapie. Berlin: Springer-Verlag; 2015

[14] Baumann K, Linden M., (Hrsg.). Weisheitstherapie. Berlin: SpringerVerlag; 2015

[15] Linden M, Schippan B, Baumann K. Weisheitstherapie-kognitive Therapie der posttraumatischen Verbitterungsstörung. Verhaltenstherapie 2004 ; 14 : 284-293

[16] Wink P, Helson R. Practical and Transcendent Wisdom: Their Nature and Some Longitudinal Findings. J Adult Dev 1997; 4: 1-14

[17] Ardelt M. Empirical assessment of a three-dimensional wisdom scale. Res Aging 2003; 25: 275-324

[18] Webster JD. An exploratory analysis of a self-assessed wisdom scale. J Adult Dev 2003; 10: 13-22

[19] Webster JD. Measuring the character strength of wisdom. Int J Aging Hum Dev 2007; 65: 163-183

[20] Staudinger UM, Glück J. Psychological Wisdom Research: Commonalities and Differences in a Growing Field. Annu Rev Psychol 2011; 62: 215-241

[21] Levenson MR, Jennings PA, Aldwin CM et al. Self-Transcendence: Conceptualization and measurement. Int J Aging Hum Dev 2005; 60 : 127-143
[22] Linden M, Ritter K. Differentielle Lebensbelastetheit. Psychiatr Psychother 2007; 4: 140-147

[23] Linden M, Rotter M, Baumann K et al. The Posttraumatic Embitterment Disorder Self-Rating Scale (PTED Scale). Clin Psychol Psychother 2009; 16: 139-147

[24] Dalbert C, Montada L, Schmitt M. Glaube an eine gerechte Welt als Motiv: Validierungskorrelate zweier Skalen. Psychologische Beiträge 1987; 29: 596-615

[25] Häder M, Häder S. Delphi und Kognitionspsychologie: ein Zugang zur theoretischen Fundierung der Delphi-Methode. ZUMA Nachrichten 1995; 19: 8-34

[26] Hasenauer M, Tschuschke V. Effektivität und Erfolgsprädiktoren in der kognitiven Verhaltenstherapie unter Praxisbedingungen. Verhaltenstherapie 2017; 27: 86-95

[27] Hellenthal A, Zimmermann P, Willmund $G$ et al. Einsatzerlebnisse, moralische Verletzungen, Werte und psychische Erkrankungen bei Einsatzsoldaten der Bundeswehr. Verhaltenstherapie 2017; 27: 244-252

[28] Linden M. Generalisierte Angststörungen. In: Batra A, Wassmann R, Buchkremer G, (Hrsg.). Verhaltenstherapie: Grundlagen-MethodenAnwendungsgebiete. Stuttgart: Thieme; 2013: 167-172

[29] Muschalla B. Arbeitsbezogene Ängste in Forschung und Praxis. Z Arb Organ 2014; 58: 206-214

[30] Berking M., (Hrsg.). Training emotionaler Kompetenzen. Berlin: Springer-Verlag; 2017

[31] Wittchen H-U, Hoyer J., (Hrsg.). Klinische Psychologie und Psychotherapie. Berlin: Springer-Verlag; 2011

[32] Heidenreich T, Michalak J. Achtsamkeit und Akzeptanz als Prinzipien in der Psychotherapie. PiD 2006; 7: 235-240

[33] Glück J, Bluck S, Baron J et al. The wisdom of experience: Autobiographical narratives across adulthood. Int J Behav Dev 2005; 29: 197-209

[34] Glück J, Baltes PB. Using the concept of wisdom to enhance the expression of wisdom knowledge: Not the philosopher's dream but differential effects of developmental preparedness. Psychol Aging 2006; 21: 679-690

[35] Bluck S, Glück J. From the inside out: people's implicit theories of wisdom. In: Sternberg R, Jordan JA, (Hrsg.). A Handbook of Wisdom: Psychological Perspectives. New York: Cambridge University Press; 2005: 84-109 